\title{
Boundary effect on multiple scattering of elastic waves in a half-space
}

\author{
A. Yu. Val'kov ${ }^{1,2}$, V. L. Kuzmin ${ }^{1,2}$, V.P. Romanov ${ }^{1}$, M. A. Nikitina ${ }^{1}$, V. Meglinskiii ${ }^{3,4}$ \\ ${ }^{1}$ Department of Physics, St. Petersburg State University, St. Petersburg, Russia \\ ${ }^{2}$ Department of Mathematics, St. Petersburg State University of Commerce \\ and Economics, St. Petersburg, Russia \\ ${ }^{3}$ Department of Physics, University of Otago, Dunedin, New Zealand \\ ${ }^{4}$ Faculty of Physics, Saratov State University, Saratov, Russia \\ alexvalk@mail.ru,kuzmin_vl@mail.ru
}

PACS 43.35.Cg, 43.20.Gp, 43.20.Bi, 43.40.Ph

DOI 10.17586/2220-8054-2015-6-4-524-536

Received: 20 June 2015

The scattering of elastic waves is studied in the vicinity of a vacuum-medium boundary. The Green's function for a half-space is re-derived within the mixed 2D-Fourier representation, which is convenient for studying layered media. Monte-Carlo simulations of elastic wave scattering from random inhomogeneities within a simplified scalar model are performed, accounting for a boundary-induced term in the Green's function. The multiply scattered elastic waves' radiation is shown to decay with distance from the source much slower in vicinity of boundary than in an infinite medium, due to the boundary condition requirements.

Keywords: Elastic waves, Boundary effect, Half-space, Multiple scattering, Monte-Carlo simulation.

\section{Introduction}

We consider the problem of scattering for radiation generated by a point-like source of harmonic oscillations in an elastic half-space. The field of the point-source, known as the Green's function of the wave equation, is fundamental for the theory of the single-, as well as multiple- scattering in random media ( $[1])$.

The problem of the displacement field generated by a point-like source in an elastic bounded medium has been considered for more than 150 years, beginning with [2], and [3]. This problem is nevertheless still relevant today $([4-6])$.

There is considerable interest in coda waves, which were interpreted in [7] as scattering from lithospheric heterogeneities (see, e.g., [8-11]). Great attention has been paid in the last few decades to the multiple scattering of waves of different physical natures in random media ( [12]). In particular, remarkable progress has been achieved in multiple light scattering (see, i.g., [13]). Subsequent methods elaborated for light scattering in random media were applied to the investigation of elastic field scattering, mainly for seismic issues ( $[14-20])$.

Previously, the multiple scattering of elastic waves were studied using the Monte Carlo method ( $[14,21,22])$ for a one-mode scalar model. Such a one-mode model can be justified with the elastic waves transfer equation ( [23]), which assumes the shear mode to be dominant. Realistically accounting for the mode conversions allowed the authors ( [18]) to show that the shear mode becomes dominant very rapidly in the coda. Detailed Monte Carlo simulations were performed in ( [24]), taking into account mode conversions and considering multiple scattering in an infinite space. 
In the case of an electromagnetic field, the larger share of the wave refracting at the boundary with vacuum escapes from the medium due to small reflectivity; the reflected share of radiation is on the order of several percent for most dielectric materials. In contrast, for elastic wave radiation, there occurs a total reflection at the boundary of an elastic medium with a vacuum, requiring that the radiation incident upon the boundary should be completely returned back to the medium. Thus, while the corrections to the Green's function caused by the boundary can be neglected for the light scattering, for acoustic radiation, the specific boundary contributions to the acoustic Green's function should turn out to be quite important.

In earlier simulations of multiple light scattering, we developed the semi-analytic Monte-Carlo approach ( [25]) for a scalar model based upon the Bethe-Salpeter equation, successively describing a number of correlation and coherent phenomena in random media optics. Here, we generalize this approach for the multiple scattering of elastic waves, also in the scalar one-mode approximation. We perform the elastic wave multiple scattering simulation, describing radiation transfer in the framework of a simplified Bethe-Salpeter equation, within the one-mode approximation. A crucial distinction of the present approach is that calculating the iterative expansion terms for the Bethe-Salpeter equation in scattering demands we use the exact form of the scalar Green's function for the half-space geometry, thus explicitly accounting for boundary conditions at the vacuum-elastic medium boundary. To the best of our knowledge, such an approach to the boundary effect problem in multiple scattering has not been applied before. We perform the Monte Carlo simulation for multiple scattering of waves propagated from a point-like radiation source to a point-like receiver. For comparison, we present the simulation results obtained using Green's function for an unbounded space. We found that using the exact Green's function for a half-space and the Green's function for an infinite space gave rather different results. The simulations have shown that multiply scattered radiation, obtained with proper accounting for the boundary, propagates in the vicinity of vacuum-medium boundary for much longer distances than would be the case for a simplified approach, wherein one neglects the boundary effect on the Green's function.

The paper is organized as follows: sction 2 contains general equations of elastic wave theory; section 3 presents the Green's function for a half-space within the 2D-Fourier representation; in the fourth section the Monte Carlo procedure accounting for boundary effect is described; section 5 contains results of simulations, and section 6 contains conclusions.

\section{Generals}

The elastic field, described as displacement vector $\mathbf{u}(\mathbf{r}, t)$ dependent on space $\mathbf{r}$ and time $t$ ( [26]), in an homogeneous medium satisfies the wave equation:

$$
\partial^{2} \mathbf{u} / \partial^{2} t-c_{t}^{2} \Delta \mathbf{u}+\left(c_{t}^{2}-c_{l}^{2}\right) \operatorname{grad} \operatorname{div} \mathbf{u}=\mathbf{0},
$$

where $c_{t}=\sqrt{\mu / \rho}$ and $c_{l}=\sqrt{(K+4 \mu / 3) / \rho}$ are the velocities of transverse and longitudinal elastic waves, respectively; $\rho$ is the density, $\mu$ and $K$ are the shear and the compression moduli. The boundary conditions at the vacuum-medium interface $B$ are defined as:

$$
\left.\boldsymbol{\sigma} \mathbf{n}\right|_{B}=\mathbf{0}
$$

where $\mathbf{n}$ is the normal vector to surface $B$, and $\boldsymbol{\sigma}(\mathbf{r}, t)$ is the stress tensor, with components

$$
\sigma_{i j}=(K-2 \mu / 3) \delta_{i j} \operatorname{div} \mathbf{u}+\mu\left(\frac{\partial u_{i}}{\partial r_{j}}+\frac{\partial u_{j}}{\partial r_{i}}\right) \text {. }
$$


We consider harmonic fields with the temporal dependence of the form $\propto \exp (-i \omega t)$, where $\omega$ is the frequency. For the harmonic point-source with frequency $\omega$, the displacement field is described by the tensor Green's function $\mathrm{G}\left(\mathbf{r}, \mathbf{r}^{\prime} ; \omega\right)$ which satisfies the equation:

$$
\rho\left[\left(c_{t}^{2}-c_{l}^{2}\right) \nabla \otimes \nabla-\mathrm{I}\left(\omega^{2}+c_{t}^{2} \Delta\right)\right] \mathrm{G}\left(\mathbf{r}, \mathbf{r}^{\prime}, \omega\right)=\mathrm{I} \delta\left(\mathbf{r}-\mathbf{r}^{\prime}\right),
$$

where $\mathbf{I}$ is the identity tensor, $\otimes$ denotes the tensor product, $\mathbf{r}^{\prime}$ and $\mathbf{r}$ are the source and receiver positions. Further, for brevity, we omit the argument $\omega \geqslant 0$.

For an infinite homogeneous medium, the Green's function $G\left(\mathbf{r}, \mathbf{r}^{\prime}\right)=G_{0}(\mathbf{R})$ with $\mathbf{R}=\mathbf{r}-\mathbf{r}^{\prime}$ due to translational invariance. This Green's function in the $(\mathbf{r}, \omega)$-representation is known to be (see, i.g. [4], Eq. (4.43)):

$$
\begin{aligned}
\mathrm{G}_{0}(\mathbf{R})=\frac{1}{\rho c_{t}^{2}} \frac{e^{i k_{t} R}}{4 \pi R}\left[\left(\mathrm{I}-\frac{\mathbf{R} \otimes \mathbf{R}}{R^{2}}\right)+\left(\frac{i}{k_{t} R}-\frac{1}{k_{t}^{2} R^{2}}\right)\left(\mathrm{I}-3 \frac{\mathbf{R} \otimes \mathbf{R}}{R^{2}}\right)\right]+ \\
+\frac{1}{\rho c_{l}^{2}} \frac{e^{i k_{l} R}}{4 \pi R}\left[\frac{\mathbf{R} \otimes \mathbf{R}}{R^{2}}-\left(\frac{i}{k_{l} R}-\frac{1}{k_{l}^{2} R^{2}}\right)\left(\mathrm{I}-3 \frac{\mathbf{R} \otimes \mathbf{R}}{R^{2}}\right)\right],
\end{aligned}
$$

where $k_{l}=\omega / c_{l}$ and $k_{t}=\omega / c_{t}$ are the longitudinal and transverse wave numbers respectively. The radiation condition adds to $k_{t, l}$ infinitesimal damping $\operatorname{Im}\left(k_{t, l}\right)=+i 0$. Forbidding waves to propagate from infinity to the source requires that the Green's function should satisfy the Sommerfield radiation condition. As can easily be seen, the near-field asymptotics $\mathrm{G}_{0}(\mathbf{R}) \propto$ $R^{-1}$, whereas terms of the form $R^{-3}$ and $R^{-2}$ cancel each other out in the short distance limit, $R \rightarrow 0$, in contrast with the electromagnetic field, containing ( [27]) asymptotic term $R^{-3}$.

For a half-space medium, the 2D Fourier transform over transversal variables $x$, $y$ turns to be an effective method for solution of the wave equation due to its cylindrical symmetry $([4,28])$. Thus, in this $\left(\mathbf{q}_{\perp}, z\right)$-representation, the wave equation (1) for temporally harmonic displacement takes the form:

$$
\left(\mathrm{K}_{2} \partial^{2} / \partial z^{2}+i \mathrm{~K}_{1} \partial / \partial z+\mathrm{K}_{0}\right) \mathbf{u}\left(\mathbf{q}_{\perp}, z\right)=\mathbf{0}
$$

where $\mathrm{K}_{2}, \mathrm{~K}_{1}=\mathrm{K}_{1}\left(\mathbf{q}_{\perp}\right)$ and $\mathrm{K}_{0}=\mathrm{K}_{0}\left(\mathbf{q}_{\perp}\right)$ are the $3 \times 3$-matrices,

$$
\begin{aligned}
& \mathrm{K}_{0}=\left(\omega^{2}-c_{t}^{2} q_{\perp}^{2}\right) \mathbf{I}-\left(c_{l}^{2}-c_{t}^{2}\right) \mathbf{Q} \otimes \mathbf{Q}, \mathrm{K}_{1}=\left(c_{l}^{2}-c_{t}^{2}\right)(\mathbf{Q} \otimes \mathbf{n}+\mathbf{n} \otimes \mathbf{Q}), \\
& \mathrm{K}_{2}=c_{t}^{2} \mathbf{I}+\left(c_{l}^{2}-c_{t}^{2}\right) \mathbf{n} \otimes \mathbf{n},
\end{aligned}
$$

and the transversal vector $\mathbf{q}_{\perp}$ is presented formally as the 3 D-vector $\mathbf{Q}=\left(\mathbf{q}_{\perp}, 0\right)$.

There are six independent solutions of Eq. (6):

$$
\mathbf{u}_{j}^{ \pm}\left(\mathbf{q}_{\perp}, z\right)=\mathbf{e}_{j}^{ \pm}\left(\mathbf{q}_{\perp}\right) e^{ \pm i \varkappa_{j}\left(q_{\perp}\right) z}, j=1,2,3,
$$

where $\varkappa_{1,2}\left(q_{\perp}\right)=\varkappa_{t}\left(q_{\perp}\right), \varkappa_{3}\left(q_{\perp}\right)=\varkappa_{l}\left(q_{\perp}\right)$,

$$
\varkappa_{t}=\sqrt{k_{t}^{2}-q_{\perp}^{2}}, \varkappa_{l}=\sqrt{k_{l}^{2}-q_{\perp}^{2}},
$$

are the eigenvalues and

$$
\begin{aligned}
& \mathbf{e}_{1}^{ \pm}\left(\mathbf{q}_{\perp}\right)=\mathbf{q}_{\perp} \times \mathbf{n} / q_{\perp}, \mathbf{e}_{3}^{ \pm}\left(\mathbf{q}_{\perp}\right)=\mathbf{q}_{l}^{ \pm}\left(q_{\perp}^{2}+\left|\varkappa_{l}\right|^{2}\right)^{-1 / 2} \\
& \mathbf{e}_{2}^{ \pm}\left(\mathbf{q}_{\perp}\right)=\left( \pm \varkappa_{t}\left(q_{\perp}\right) \mathbf{q}_{\perp} / q_{\perp}-q_{\perp} \mathbf{n}\right)\left(q_{\perp}^{2}+\left|\varkappa_{t}\right|^{2}\right)^{-1 / 2}
\end{aligned}
$$

- eigenvectors of the second order matrix differential operator in Eq. (6), with

$$
\mathbf{q}_{l}^{ \pm}=\left(\mathbf{q}_{\perp}, \pm \varkappa_{l}\left(q_{\perp}\right)\right)
$$


being the 3D wave vector. The waves with superscripts " \pm " propagate along axis $z$ in directions $z \rightarrow \pm \infty$, respectively. These normal waves have simple physical interpretation: $\mathbf{u}_{1,2}^{ \pm}\left(\mathbf{q}_{\perp}, z\right)$ are the transverse waves with wave vectors $\mathbf{q}_{t}^{ \pm}$and $\mathbf{u}_{3}^{ \pm}\left(\mathbf{q}_{\perp}, z\right)$ are the longitudinal waves with wave vectors $\mathbf{q}_{l}^{ \pm}$. Unit vectors $\mathbf{e}_{j}^{ \pm}$are the polarizations of these waves.

It should be noted that due to the equality $\varkappa_{1}=\varkappa_{2}$, one can take as a set of eigenvectors $\mathbf{e}_{1,2}^{ \pm}$any pair of linear independent vector satisfying to the orthogonality condition $\mathbf{e}_{1,2}^{ \pm} \perp \mathbf{q}_{t}^{ \pm}$. We have fixed the choice requiring supplementarily the orthogonality relations $\mathbf{e}_{1}^{ \pm} \perp \mathbf{n}, \mathbf{e}_{1}^{ \pm} \perp \mathbf{q}_{\perp}$ and $\mathbf{e}_{1}^{ \pm} \perp \mathbf{e}_{2}^{ \pm}$should be fulfilled. Then, the subscript $j=1$ corresponds to $\mathrm{SH}$-wave, $j=2$ - to SV-wave, and $j=3$ - to the P-wave.

\section{The Green's function in a Half-Space}

Let an elastic medium occupy the half-space $z>0$. There have been several derivations beside the classic ones for the Greens function in an elastic half-space; our approach is quite close to that used by Johnson. The Green's function within the $\left(\mathbf{q}_{\perp}, z\right)$-representation with the $\delta$-form source obeys the equation:

$$
\rho\left(\mathrm{K}_{2} \partial^{2} / \partial z^{2}+i \mathrm{~K}_{1} \partial / \partial z+\mathrm{K}_{0}\right) \mathrm{G}\left(\mathbf{q}_{\perp} ; z, z^{\prime}\right)=\mathrm{I} \delta\left(z-z^{\prime}\right)
$$

The solution of Eq. (12) supplemented with boundary conditions at $z=0$ can be sought as a sum of two terms

$$
\mathrm{G}\left(\mathbf{q}_{\perp} ; z, z^{\prime}\right)=\mathrm{G}_{0}\left(\mathbf{q}_{\perp} ; z-z^{\prime}\right)+\mathrm{G}_{B}\left(\mathbf{q}_{\perp} ; z, z^{\prime}\right)
$$

where $G_{0}$ is the Green's function for the infinite homogenous medium and $G_{B}$, being the solution of the homogeneous wave equation

$$
\left(\mathrm{K}_{2} \partial^{2} / \partial z^{2}+i \mathrm{~K}_{1} \partial / \partial z+\mathrm{K}_{0}\right) \mathbf{G}_{B}\left(\mathbf{q}_{\perp} ; z, z^{\prime}\right)=\mathbf{0}
$$

satisfies to the boundary condition.

The first term, the Green's function for an infinite homogeneous medium (5), can be written in the form:

$$
\mathbf{G}_{0}\left(\mathbf{q}_{\perp}, z-z^{\prime}\right)=i\left(2 \varkappa_{t} \rho \omega^{2}\right)^{-1}\left[\varkappa_{t}^{-1} e^{i \varkappa_{t}\left|z-z^{\prime}\right|}\left(k_{t}^{2} \mathbf{I}-\mathbf{q}_{t}^{ \pm} \otimes \mathbf{q}_{t}^{ \pm}\right)+\varkappa_{l}^{-1} e^{i \varkappa_{l}\left|z-z^{\prime}\right|} \mathbf{q}_{l}^{ \pm} \otimes \mathbf{q}_{l}^{ \pm}\right]
$$

where superscripts "+" must be chosen for $z-z^{\prime}>0$ and "-" for $z-z^{\prime}<0$ in $\mathbf{q}_{t, l}^{ \pm}$.

Since components of the tensor Green's function can be interpreted as matter displacements, induced by the point source, the boundary conditions (2) give:

$$
\left.\left(\mathrm{B}_{1} \partial / \partial z+i \mathrm{~B}_{0}\right) \mathrm{G}\left(\mathbf{q}_{\perp} ; z, z^{\prime}\right)\right|_{z=0}=\mathbf{0},
$$

where $z^{\prime}$ is an arbitrary point inside the medium, $z^{\prime}>0$,

$$
\mathrm{B}_{0}=\mu \mathbf{Q} \otimes \mathbf{n}+(K-2 \mu / 3) \mathbf{n} \otimes \mathbf{Q}, \mathbf{B}_{1}=\mu \mathbf{l}+(K+\mu / 3) \mathbf{n} \otimes \mathbf{n} .
$$

Additionally, one should require there should be no wave propagation from infinity, $+\infty$, to the source. Since, for the body term $\mathrm{G}_{0}\left(\mathbf{q}_{\perp} ; z, z^{\prime}\right)$, this requirement is fulfilled, it should also be fulfilled for the term $\mathrm{G}_{B}\left(\mathbf{q}_{\perp} ; z, z^{\prime}\right)$.

The solution for matrix equation (14), containing no wave propagation from infinity, can be written as:

$$
\mathbf{G}_{B}\left(\mathbf{q}_{\perp} ; z, z^{\prime}\right)=\sum_{j, m=1}^{3} C_{j m} \mathbf{u}_{m}^{+}\left(\mathbf{q}_{\perp} ; z\right) \otimes \mathbf{u}_{j}^{-}\left(\mathbf{q}_{\perp} ;-z^{\prime}\right),
$$

where $C_{j m}=C_{j m}\left(\mathbf{q}_{\perp}\right)$ are the coefficients determined by the set of boundary conditions (16),

$$
\left.\left(\mathrm{B}_{1} \partial / \partial z+i \mathrm{~B}_{0}\right) \mathrm{G}_{B}\left(\mathbf{q}_{\perp} ; z, z^{\prime}\right)\right|_{z=0}=-\left.\left(\mathrm{B}_{1} \partial / \partial z+i \mathrm{~B}_{0}\right) \mathrm{G}_{0}\left(\mathbf{q}_{\perp} ; z, z^{\prime}\right)\right|_{z=0}
$$


Physically, the $(j, m)$ term describes wave $\mathbf{u}_{j}^{-}$incident upon the boundary and reflected as wave $\mathbf{u}_{m}^{+}$, propagating from boundary to receiver. We schematically illustrate in Fig. 1 the propagation of waves, constituting the Green's function $\mathrm{G}\left(\mathbf{r}^{\prime}, \mathbf{r}\right)$, in a half space with boundary $B$, from the source to receiver.

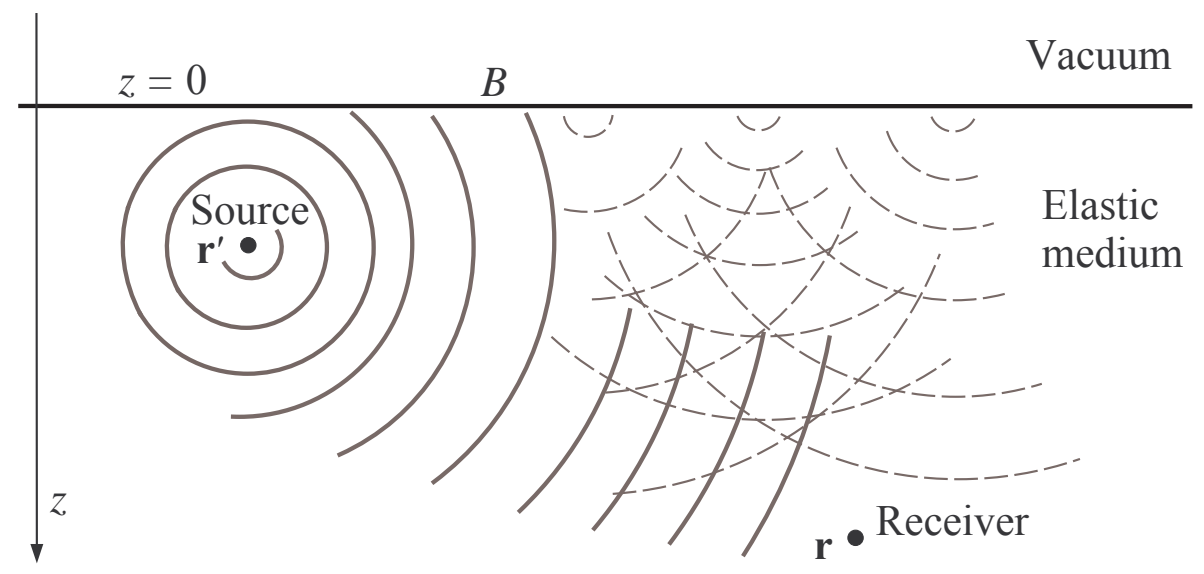

FIG. 1. Schematic source and receiver arrangement in the half-space geometry. The solid and dash circular arcs represent waves $\mathrm{G}_{0}$ and $\mathrm{G}_{B}$, respectively, and $B$ is the boundary of the medium-vacuum.

Substituting Eqs. (18) and (15) into (19), we obtain a set of algebraic equations for nine coefficients $C_{j m}$ with solution in the form:

$$
C_{j m}=-i a_{m j}\left(2 \rho c_{j}^{2} \varkappa_{j} D\right)^{-1}
$$

where $j, m=1-3 ; c_{1,2}=c_{t}, c_{3}=c_{l}$, and

$$
\begin{aligned}
& a_{11}=D, D=4 \varkappa_{t} \varkappa_{l} q_{\perp}^{2}+E^{2}, E=k_{t}^{2}-2 q_{\perp}^{2}, a_{22}=a_{33}=D-2 E^{2}, \\
& a_{23}=-4 c_{l} c_{t}^{-1} \varkappa_{l} q_{\perp} E, a_{32}=-a_{23} c_{t}^{2} c_{l}^{-2} \varkappa_{t} \varkappa_{l}^{-1}, a_{1 j}=a_{j 1}=0(j=2,3) .
\end{aligned}
$$

Performing the inverse Fourier transform we present the boundary induced term $\mathrm{G}_{B}$ in the physical space as follows:

$$
\mathbf{G}_{B}\left(\mathbf{R}_{\perp} ; z, z^{\prime}\right)=-\frac{i}{2 \rho} \sum_{j, m=1}^{3} c_{j}^{-2} \int \frac{\mathrm{d} \mathbf{q}_{\perp}}{(2 \pi)^{2}} \frac{a_{m j}}{D \varkappa_{j}} e^{i \mathbf{q}_{\perp} \cdot \mathbf{R}_{\perp}} e^{i\left(\varkappa_{m} z+\varkappa_{j} z^{\prime}\right)} \mathbf{e}_{m}^{+}\left(\mathbf{q}_{\perp}\right) \otimes \mathbf{e}_{j}^{-}\left(\mathbf{q}_{\perp}\right) .
$$

The 2D-integrals are expressed through 1D-integrals for the Bessel functions. The $(j, m)$ term in this sum can be described as an input for the transformation of the $j$-mode incident wave into the $m$-mode generated wave. The zero values for coefficients $a_{12}, a_{21}, a_{13}, a_{31}$ correspond to the well-known selection rules ( $[4])$.

Various contributions to integrals $(22)$ over $\mathbf{q}_{\perp}$ reproduce the known types of elastic waves of different physical nature. The vicinity of stationary phases in exponentials of Eq. (22) yields the known formulas of geometrical acoustics describing all pairs of different modes of incident and reflected waves. The pole singularity due to the zero of determinant $D\left(q_{\perp}\right)$ in the integrand, gives rise to surface Rayleigh waves. The zeros of $\varkappa_{j}$ at $q_{\perp}=k_{t, l}$, being the branch points of the integrands, give rise to head waves.

In the far-field zone, $q_{\perp} R_{\perp}+z \varkappa_{m}+z^{\prime} \varkappa_{j} \gg 1$, the integrands in Eq. (22) contain rapidly oscillating exponents and the method of stationary phase can be applied. The relevant 
contribution to each integral comes from the range of the stationary point $\mathbf{q}_{\mathrm{st}}^{j m}=q_{j m} \mathbf{R}_{\perp} / R_{\perp}$, $\left(0 \leqslant q_{j m}<k_{t, l}\right)$, which can be found as the root of equation:

$$
z^{\prime} q_{j m} \varkappa_{j}^{-1}\left(q_{j m}\right)+z q_{j m} \varkappa_{m}^{-1}\left(q_{j m}\right)=R_{\perp} .
$$

As a result, this contribution can be presented as follows:

$$
\mathbf{G}_{B}^{\mathrm{st}}\left(\mathbf{R}_{\perp} ; z, z^{\prime}\right) \sim-\sum_{j, m=1}^{3} \frac{1}{\rho c_{j}^{2}} F_{j m}\left(q_{j m}\right) \frac{e^{i \Phi_{j m}\left(q_{j m}\right)}}{4 \pi R_{j m}\left(q_{j m}\right)} \mathbf{e}_{m}^{+}\left(\mathbf{q}_{\mathrm{st}}^{j m}\right) \otimes \mathbf{e}_{j}^{-}\left(\mathbf{q}_{\mathrm{st}}^{j m}\right),
$$

where

$$
R_{j m}=\sqrt{\left(R_{\mathrm{in}}^{j} k_{j}+R_{\mathrm{ref}}^{m} k_{m} \varkappa_{j}^{2} \varkappa_{m}^{-2}\right) R_{\perp} q_{j m}^{-1}},
$$

and $R_{\text {in }}^{j}\left(q_{j m}\right)$ and $R_{\text {ref }}^{m}\left(q_{j m}\right)$ can be interpreted as the geometric acoustic path of an elastic wave traveling from the source to the boundary in the form of the $j$-th mode and reflected from the boundary to the receiver as the $m$-th mode, and:

$$
\Phi_{j m}=q_{j m} R_{\perp}+\varkappa_{m}\left(q_{j m}\right) z+\varkappa_{j}\left(q_{j m}\right) z^{\prime}
$$

is the total phase, and $F_{j m}\left(q_{j m}\right)=a_{m j}\left(q_{j m}\right) / D\left(q_{j m}\right)$ are the generalized reflection coefficients. Coefficient $F_{j m}$ describes conversion of the $j$-th incident mode into the $m$-th reflected mode at the medium-vacuum boundary. It also accounts for phase shifts and the solid angle transformation occurring at reflection of spherical waves (see Sec. 6 in [4], cf. in optics [29, $30])$.

It should be noted that the terms of asymptotic formula (24) can be interpreted as five spherical waves generated by three imaginary sources.

The near-field zone asymptotics of the Green's function, described by (22), as well as the Rayleigh wave contribution, were considered within the approach outlined in [31].

There have been several derivations for the Green's function in an elastic half-space, however, our approach is quite similar to that used in ( [28]).

\section{Multiple scattering in a half-space}

In addition to the coherent wave propagating from a source in a heterogeneous elastic medium, scattered waves also appear. The radiation is scattered from fluctuations of density $\rho^{(\mathrm{fl})}(\mathbf{r})$ as well as material parameters, which are generally described with the fourth rank tensor $\mathrm{C}^{(\mathrm{fl})}(\mathbf{r})$. For a locally-isotropic medium, it can be written as:

$$
C_{\alpha \beta \gamma \zeta}^{(\mathrm{fl})}(\mathbf{r})=K^{(\mathrm{fl})}(\mathbf{r}) \delta_{\alpha \beta} \delta_{\gamma \zeta}+\mu^{(\mathrm{fl})}(\mathbf{r})\left(\delta_{\alpha \gamma} \delta_{\beta \zeta}+\delta_{\alpha \zeta} \delta_{\gamma \beta}-\frac{2}{3} \delta_{\alpha \beta} \delta_{\gamma \zeta}\right),
$$

where $K^{(\mathrm{f})}(\mathbf{r})$ and $\mu^{(\mathrm{fl})}(\mathbf{r})$ are the random, or fluctuating compression and shear modules.

We present the material parameters as

$$
\rho^{(\mathrm{fl})}(\mathbf{r})=\rho+\delta \rho(\mathbf{r}), \mathrm{C}^{(\mathrm{fl})}(\mathbf{r})=\mathrm{C}+\delta \mathrm{C}(\mathbf{r}),
$$

where $\delta \rho(\mathbf{r})$ and $\delta \mathrm{C}(\mathbf{r})$ are random fluctuations with respect to the average values for $\rho$ and C.

The wave equation for the Green's function $\mathrm{G}^{(\mathrm{f})}$ in a random medium in the $(\mathbf{r}, \omega)$ representation can be written as:

$$
\left[\rho^{(\mathrm{f})}(\mathbf{r}) \delta_{\alpha \gamma} \omega^{2}+\frac{\partial}{\partial r_{\beta}}\left(C_{\alpha \beta \gamma \zeta}^{(\mathrm{fl})}(\mathbf{r}) \frac{\partial}{\partial r_{\zeta}}\right)\right] G_{\gamma \eta}^{(\mathrm{fl})}\left(\mathbf{r}, \mathbf{r}^{\prime}\right)=-\delta_{\alpha \eta} \delta\left(\mathbf{r}-\mathbf{r}^{\prime}\right) .
$$


For an homogeneous non-fluctuating medium, it returns to (4). The differential equation (29) can be written in the form of an integral equation (see [32]):

$$
\begin{aligned}
\mathrm{G}^{(\mathrm{fl})}\left(\mathbf{r}, \mathbf{r}^{\prime}\right)=\mathrm{G}\left(\mathbf{r}, \mathbf{r}^{\prime}\right)+\omega^{2} \int \mathrm{G}\left(\mathbf{r}, \mathbf{r}_{1}\right) \delta \rho\left(\mathbf{r}_{1}\right) \mathrm{G}^{(\mathrm{fl})}\left(\mathbf{r}_{1}, \mathbf{r}^{\prime}\right) \mathrm{d} \mathbf{r}_{1}- & \\
& -\int \frac{\partial \mathrm{G}\left(\mathbf{r}, \mathbf{r}_{1}\right)}{\partial \mathbf{r}_{1}} \delta \mathrm{C}\left(\mathbf{r}_{1}\right) \frac{\partial \mathrm{G}^{(\mathrm{fl})}\left(\mathbf{r}_{1}, \mathbf{r}^{\prime}\right)}{\partial \mathbf{r}_{1}} \mathrm{~d} \mathbf{r}_{1} .
\end{aligned}
$$

Here, $\mathrm{G}\left(\mathbf{r}, \mathbf{r}^{\prime}\right)$ is the Green's function of an homogeneous medium occupying the half-space.

Our aim is to study the effect of the boundary on multiple scattering. Multiple scattering has been studied in detail for the case of an electromagnetic field for some time. However, the boundary conditions turn to be quite different for elastic or electromagnetic fields. Namely, for the case of elastic waves and a medium-vacuum boundary, the scattered radiation remains in the medium, in contrast with the electromagnetic field, which permits us to disregard a reflection at the dielectric-vacuum boundary.

In order to illustrate the influence of boundaries on the multiple scattering of elastic waves we introduce a number of simplifying assumptions.

First, we will neglect the tensorial character of the wave equation (30). Then, the Green's function for a half-space takes the form of sum of two spherical scalar waves:

$$
G\left(\mathbf{r}, \mathbf{r}^{\prime}\right)=G_{0}\left(\mathbf{r}-\mathbf{r}^{\prime}\right)+G_{B}\left(\mathbf{r} ; \mathbf{r}^{\prime}\right)
$$

here, the first term is the field of the point-source in an infinite medium:

$$
G_{0}\left(\mathbf{r}-\mathbf{r}^{\prime}\right)=1 /\left(4 \pi \rho c^{2}\right)\left|\mathbf{r}-\mathbf{r}^{\prime}\right|^{-1} \exp \left(i k\left|\mathbf{r}-\mathbf{r}^{\prime}\right|\right)
$$

and the second one:

$$
G_{B}\left(\mathbf{r} ; \mathbf{r}^{\prime}\right)=-G_{0}\left(\mathbf{r}-\mathbf{r}_{M}^{\prime}\right)
$$

can be interpreted as the field of the source's mirror image, located at point $\mathbf{r}_{M}^{\prime}=\left(x^{\prime}, y^{\prime},-z^{\prime}\right)$ where $\mathbf{r}^{\prime}=\left(x^{\prime}, y^{\prime}, z^{\prime}\right)$ and $c$ is the velocity of the considered wave mode. (Formally the tensor equations (13), (5) and (24) are reduced to scalar ones, (31)-(33), if $c_{l}=c_{t}=c$; this condition is non-physical for an elastic medium, but simplifies greatly the mathematical aspects of multiple scattering analysis and simulation.) The wave number $k=k^{\prime}+i k^{\prime \prime}$ contains both the real part, $k^{\prime}=\omega / c$, as well as the imaginary part, $k^{\prime \prime}=1 /(2 l)$, where $l$ is the extinction length; the extinction is contributed by elastic scattering and adsorption, namely $l^{-1}=l_{a}^{-1}+l_{s}^{-1}$ where $l_{s}$ is the scattering mean free path and $l_{a}$ is adsorption length. A similar scalar model has been used in a number of Monte-Carlo simulations for seismic problems (see, e.g., [22,33]).

Secondly, we will neglect fluctuations of elastic moduli as compared with the density fluctuations. This approach corresponds to the widely-known assumption that the fluctuations of thermodynamic variable derivatives are to be smaller than those of the variables themselves; thus we neglect the second integral term in (30).

As a result, we arrive at the one-mode scalar approximation for the considered wavescattering problem. In particular, we neglect the difference in reflection for the specific modes of different types and do not consider the non-trivial transformation of the longitudinal and transverse waves under scattering. These effects are significant, and thus, require special consideration. Note also that with these simplifications, the simulation of the elastic field transfer turns to be exactly the same as simulations of light radiation in a random medium in the scalar field approach framework ( [25]). Additionally, to make the boundary conditions as distinct as possible from that of light scattering, we presently add a term accounting for the contribution of reflected radiation for every scattering event. 
The intensity of scattered radiation, $I_{\mathrm{sc}}\left(\mathbf{r}, \mathbf{r}^{\prime}\right)$, transferred from the source in point $\mathbf{r}^{\prime}$ to receiver located in $\mathbf{r}$, is proportional to the square of the Green's function modulus:

$$
I_{\mathrm{sc}}\left(\mathbf{r}, \mathbf{r}^{\prime}\right) \propto\left|G^{(\mathrm{fl})}\left(\mathbf{r}, \mathbf{r}^{\prime}\right)\right|^{2}
$$

Iterating the integral equation (30) and multiplying it by its complex conjugate, one presents the quadratic form (34) as a series in orders of scattering.

For problems dealing with multiple scattering, all the contributions wherein the phase shift between the pair of complex-conjugated fields is not compensated are known to be neglected due to the random configuration of the medium's inhomogeneities. As a result, one can restricts oneself only with ladder contributions, wherein paths traveled by a pair of complex-conjugated fields coincide. Thus, the multiple scattering intensity can be presented as the series:

$$
\begin{aligned}
& I_{\mathrm{sc}}\left(\mathbf{r}, \mathbf{r}^{\prime}\right) \propto \sum_{\sigma_{0,1}} \int \Lambda\left(\mathbf{r}, \mathbf{r}_{1}^{\sigma_{1}}\right) B\left(\mathbf{k}_{(s) 1}^{\sigma_{1}}-\mathbf{k}_{(i) 0}^{\sigma_{0}}\right) \Lambda\left(\mathbf{r}_{1}, \mathbf{r}^{\prime \sigma_{0}}\right) \mathrm{d} \mathbf{r}_{1}+ \\
& +\sum_{\sigma_{0,1,2}} \int \Lambda\left(\mathbf{r}, \mathbf{r}_{2}^{\sigma_{2}}\right) B\left(\mathbf{k}_{(s) 2}^{\sigma_{2}}-\mathbf{k}_{(i) 1}^{\sigma_{1}}\right) \Lambda\left(\mathbf{r}_{2}, \mathbf{r}_{1}^{\sigma_{1}}\right) B\left(\mathbf{k}_{(s) 1}^{\sigma_{1}}-\mathbf{k}_{(i) 0}^{\sigma_{0}}\right) \Lambda\left(\mathbf{r}_{1}, \mathbf{r}^{\sigma_{0}}\right) \mathrm{d} \mathbf{r}_{1} \mathrm{~d} \mathbf{r}_{2}+\ldots,
\end{aligned}
$$

where the propagator in an infinite medium $\Lambda\left(\mathbf{R}, \mathbf{R}^{\prime}\right)$ describing the radiation transfer between two successive scattering events occurring at points $\mathbf{r}_{j+1}$ and $\mathbf{r}_{j}$ is formed by the product of pair of the Green's function (31)

$$
\Lambda\left(\mathbf{r}_{j+1}, \mathbf{r}_{j}^{\sigma}\right)=\left|G_{0}\left(\mathbf{r}_{j+1}-\mathbf{r}_{j}^{\sigma}\right)\right|^{2}
$$

The cross-terms $G_{0} G_{B}^{*}, G_{0}^{*} G_{B}$ are omitted due to chaotic randomization of phase shifts over the inhomogeneities.

Subscripts $j=1,2, \ldots, n$ enumerate the scattering events, therewith indices $j=0$ and $j=n+1$ refer to the source and receiver $, \mathbf{r}_{0}=\mathbf{r}^{\prime}, \mathbf{r}_{n+1}=\mathbf{r}$, respectively, (Fig. 2a);

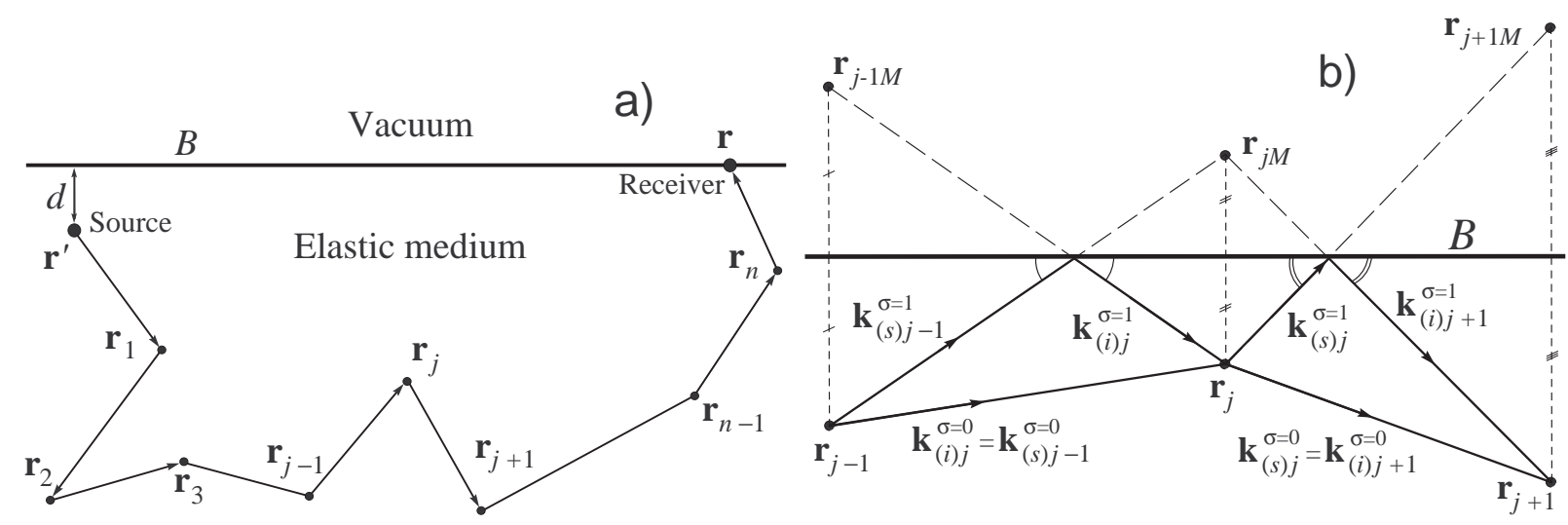

FIG. 2. a) The stochastic trajectory presented as the path traveled by a "phonon packet" undergoing a sequence of $n$ scattering events. b) Wave vectors of incident, $\mathbf{k}_{(i) j}^{\sigma}$, and scattered, $\mathbf{k}_{(s) j}^{\sigma}$, elastic waves involved in the $j$-th scattering event. Superscripts $\sigma=0$ and $\sigma=1$ correspond to the direct propagation of the beam and to propagation with intermediate reflection, respectively.

$$
\mathbf{r}_{j}^{\sigma}= \begin{cases}\mathbf{r}_{j}, & \sigma=0 \\ \mathbf{r}_{j M}, & \sigma=1\end{cases}
$$


points $\mathbf{r}_{j}$ and $\mathbf{r}_{j M}$ are the positions of the $j$-th scatterer and its mirror image, $\mathbf{r}_{0 M} \mathbf{r}_{n+1, M}$ are the mirror images of the source and the receiver (Fig. 2b). Wave vectors of incident, $\mathbf{k}_{(i) j}^{\sigma}$, and scattered, $\mathbf{k}_{(s) j}^{\sigma}$, elastic waves in $j$-th scattering event are

$$
\mathbf{k}_{(i) j}^{\sigma}=k^{\prime}\left(\mathbf{r}_{j}-\mathbf{r}_{j-1}^{\sigma}\right) /\left|\mathbf{r}_{j}-\mathbf{r}_{j-1}^{\sigma}\right|, \mathbf{k}_{(s) j}^{\sigma}=k^{\prime}\left(\mathbf{r}_{j+1}^{\sigma}-\mathbf{r}_{j}\right) /\left|\mathbf{r}_{j+1}^{\sigma}-\mathbf{r}_{j}\right| .
$$

Summing over $\sigma_{0}, \sigma_{1}, \ldots$ in Eq. (35), we account for reflections of incident and scattered beams of all scattering orders. Thus, accounting for the boundary effect doubles the number of terms of Eq. (35) with the scattering order increasing per unit.

The phase function $B(\mathbf{q})$ is the Fourier transform of the density correlation function

$$
B(\mathbf{q})=\int\left\langle\delta \rho(\mathbf{R}) \delta \rho\left(\mathbf{R}^{\prime}\right)\right\rangle e^{-i \mathbf{q}\left(\mathbf{R}-\mathbf{R}^{\prime}\right)} \mathrm{d} \mathbf{R}
$$

where $\mathbf{q}$ is the momentum transfer $\mathbf{q}=\mathbf{k}_{(s)}-\mathbf{k}_{(i)}$. For the isotropic scattering phase function, $B(q)$ is constant; for anisotropic scattering, it depends on the scattering angle $\theta$ through wave vectors of incidence $\mathbf{k}_{(i)}$ and $\mathbf{k}_{(s)}, q=2 k^{\prime} \sin (\theta / 2)$.

Accounting for the explicit form of the Green's function of a scalar field for a halfspace (31), (32), the propagator, describing the radiation transfer between two successive events of scattering in Eq. (35), can be written as follows:

$$
\Lambda\left(\mathbf{r}_{j+1}, \mathbf{r}_{j}^{\sigma}\right)=\left(4 \pi \rho c^{2}\left|\mathbf{r}_{j+1}-\mathbf{r}_{j}^{\sigma}\right|\right)^{-2} \exp \left(-\left|\mathbf{r}_{j+1}-\mathbf{r}_{j}^{\sigma}\right| / l\right)
$$

The exponential decay factor comes from the imaginary part of the wave number in Eq. (32).

Within the Monte-Carlo method, one simulates a stochastic trajectory (Fig. 2). The average over these trajectories gives the scattered radiation intensity.

A random trajectory is constructed recurrently. Let $\mathbf{r}_{j}$ be the position of the $j$-th scattering event. We define the position of the next scattering event as $\mathbf{r}_{j+1}=\mathbf{r}_{j}+\mathbf{r}$. Let $U_{1}$, $U_{2}$, and $U_{3}$ be three independent random variables uniformly distributed within the interval $(0 ; 1)$; one determines spherical coordinates of vector $\mathbf{r}$ with $\mathbf{r}_{j}$ as polar axis, namely the distance $r=|\mathbf{r}|$, and polar and azimuthal angles, $\theta$ and $\phi$, via these random variables, as follows.

The distance $r=\left|\mathbf{r}_{j+1}-\mathbf{r}_{j}^{\sigma}\right|$ is generated using the substitution $r=-l \ln U_{1}$. In this way, the inverse transform sampling method is realized (see, i.g., [34]) which takes into account the exponential decay in Eq. (39).

The azimuthal $\phi$ is distributed uniformly, $\phi=2 \pi U_{2}$.

The angle $\theta$ in the own coordinate frame for vector $\mathbf{r}_{j}$ is generated using the substitution $\theta=F^{-1}\left(U_{3}\right)$, where $F^{-1}$ is the inverse transform of function:

$$
F(x)=\left(\int_{0}^{\pi} B\left(2 k^{\prime} \sin (\theta / 2)\right) \mathrm{d} \theta\right)^{-1} \int_{0}^{\pi x} B\left(2 k^{\prime} \sin (\theta / 2)\right) \mathrm{d} \theta .
$$

The $(j+1)$-th scatterer position $\mathbf{r}_{j+1}$ and superscript $\sigma_{j}$ are defined as follows. If the point $\mathbf{r}_{j}+r \mathbf{n}$ is located inside the elastic half space (i.e. $r_{j z}+r n_{z} \geqslant 0$ ) then $\mathbf{r}_{j+1}=\mathbf{r}_{j}+r \mathbf{n}$ and we take $\sigma_{j}=0$. Otherwise, the point $\mathbf{r}_{j}+r \mathbf{n}$ is reflected: $\mathbf{r}_{j+1}=\left(\mathbf{r}_{j}+r \mathbf{n}\right)_{M}$ and we take $\sigma_{j}=1$.

The number of scattering events, $n_{\max }$, are determined with the requirement that the numerical data become stable. In our simulations $n_{\max }$ did not exceed several hundreds. 


\section{Results of simulation}

We performed the Monte-Carlo simulation for elastic radiation transfer in the vicinity of a boundary with vacuum for a scalar scattering model for distances far exceeding the characteristic extinction. The approach based on the radiative transfer theory requires that the wavelength should be small, as compared to the extinction length. Performing calculations, we take the sound velocity as $c=2.5 \times 10^{3} \mathrm{~m} / \mathrm{s}$, and the radiation frequency as $f=10^{2} \mathrm{~Hz}$, which produces the wavelength of $\lambda=25 \mathrm{~m}$. We then take the extinction length, range as $l=200 \mathrm{~m}$ to $l=1000 \mathrm{~m}$.

To account for scattering anisotropy, we apply the Henyey-Greenstein phase function

$$
B\left(2 k^{\prime} \sin (\theta / 2)\right) \propto\left(1-g^{2}\right)\left(1+g^{2}-2 g \cos \theta\right)^{-3 / 2} .
$$

containing the unique parameter, the mean cosine of scattering angle, $g=\langle\cos \theta\rangle$. This function, widely used in many radiation transfer problems ( [1]), is a particular realization of the von Kármán phase function (see for example [35]):

$$
B(q) \propto\left(1+q^{2} a^{2}\right)^{-3 / 2-\varkappa},
$$

well-known in geophysics, with the Hurst exponent $\varkappa=0$ and characteristic size of inhomogeneities $a$ being specified as $a=k^{-1} \sqrt{g} /(1-g)$.

We have also used the optical theorem (see, e.g., $[36,37]$ ) which relates the extinction length $l$ and phase function $B(q)$.

We have shown the intensity of multiply scattered radiation as a function of the distance between the source and receiver. We present results for two positions of the source, either at the surface, or at one kilometer deep $d$ in the medium; the receiver is placed at the boundary.

In Figs. 3a and 3b, the presented results for scattered intensity calculations were shown to be dependent on the distance between the source and the receiver. In Fig. 3a results are presented for isotropic scattering, $g=0$, and in Fig. $3 \mathrm{~b}$ - for anisotropic scattering, $g=0.8$.

For isotropic scattering, the plots of intensity for multiply-scattered radiation, shown in the same units as function of the source-receiver distance, turn out to be quite close for both geometries. For small distances it can be explained by equal values for the ballistic "phonon" inputs which are dominant at this spatial range, while with increased distances, the depth of the source positions becomes negligible in comparison to the distance along the surface.

For anisotropic scattering, the picture is different. The intensity of multiply scattered radiation turns to be noticeably smaller for the geometry with an imbedded source, as compared to the surface-based source. For comparison, we show the simulation results obtained for the model wherein the boundary of the medium is accounted for only in the spatial integrals over half-space in the series over the scattering orders obtained by iterations of integral equations like the Bethe-Salpeter equation Eq. (30). Such an approach was specifically used in $[14,15]$. The principal distinction of our approach is that we not only account for the boundedness of the scattering medium by performing the spatial integrations, but also the fact that the integrand itself, being the pair product of Green's functions, varies approaching the boundary range. For both geometries, the radiation intensity is seen to be smaller for isotropic as well as anisotropic scattering, and decays with distance much faster. Physically, this can be explained by the loss of "phonons" at the boundary due to the neglect of reflection. 

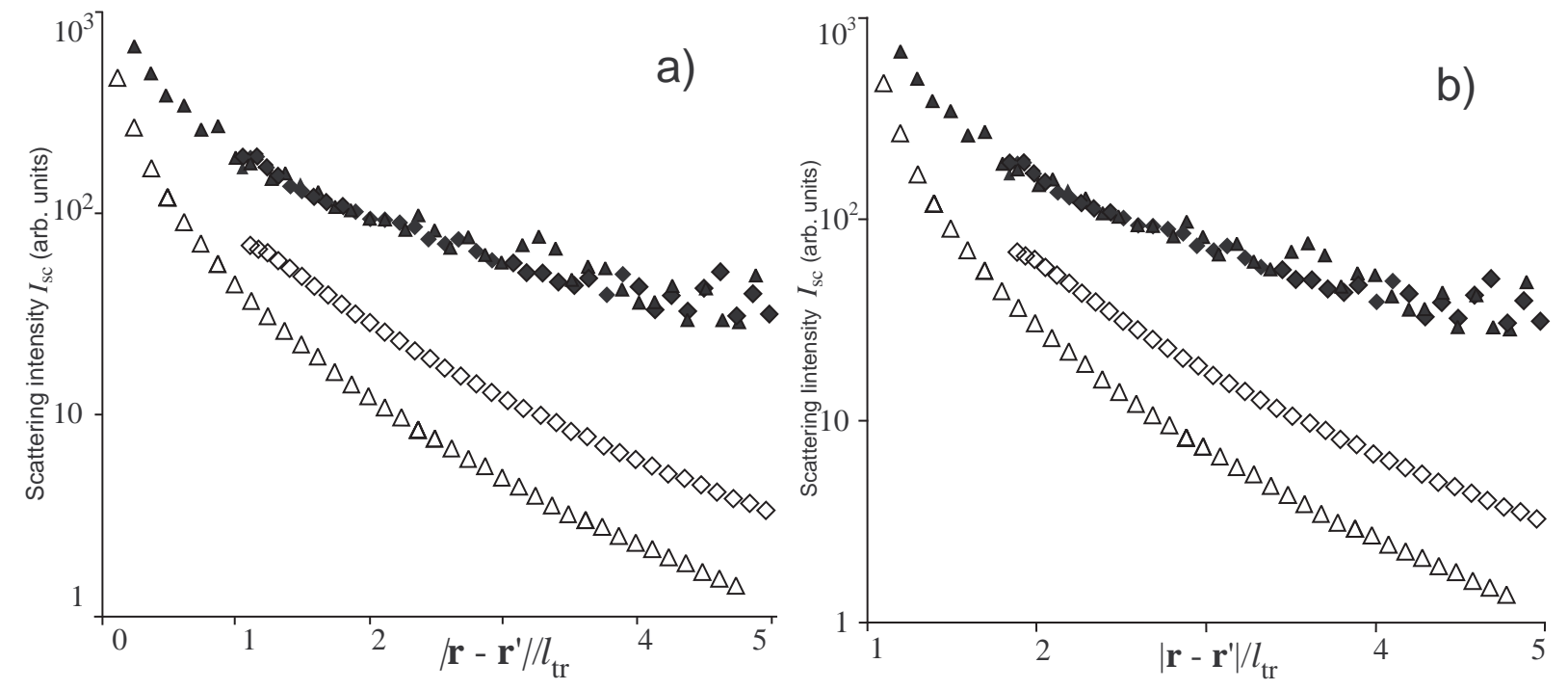

FIG. 3. Intensity of multiply scattered radiation via distance between source and receiver. The sampling volume $N=2 \times 10^{6}$. The receiver moves along the surface, and the source is located: $\boldsymbol{\Delta}$ and $\Delta$ - at the surface, $\downarrow$ and $\diamond-$ at the depth of $1 \mathrm{~km}$; For both plots: $\triangle$ - and $\diamond-$ the reflection of radiation at the boundary is neglected thoroughly, for $\boldsymbol{\Delta}$ and $\boldsymbol{\nabla}$ the total reflection at the boundary is supposed. a) isotropic scattering, $g=0$, scattering mean free path $l_{s}=1000 \mathrm{~m}$. b) anisotropic scattering, $g=0.8$, scattering mean free path $l_{s}=200 \mathrm{~m}$, adsorption length $l_{a}=10000 \mathrm{~km}$ for both figures.

In media with an anisotropic scattering indicatrix, the transport length $l_{\operatorname{tr}}=l_{s}(1-$ $\langle\cos \theta\rangle)^{-1}$, is known ( [1]) to take part of the universal spatial scale for multiple scattering instead of the scattering mean free path $l_{s}$. Thus, we plot the distance between source and receiver in units of $l_{\mathrm{tr}}$. This permits us to compare results for simulations of acoustic as well as optical models with quite different spatial dimensions.

This effect is especially pronounced within the boundary region, when the source and receiver are both near the surface. It should be noted that the curves, plotted in terms of transport length, would appear to be quite non-sensitive to anisotropy of the scattering cross-section. This verifies the widely acknowledged assumption regarding the validity of the diffusion mechanism for radiation transfer independently of the physical nature of radiation.

\section{Conclusion}

Thus, numerical simulations performed for a quite simplified model of an elastic medium exhibit the important role boundary conditions play in the description of multiple scattering; we have shown that proper accounting for the boundary requires that the form of the Green's function itself should be modified due to the boundary conditions.

Simulating the elastic random wave transfer, we have used a previously-developed approach for the study of multiple scattering of light in random media. However, the boundary conditions for elastic waves highlight a fundamental distinction from that of light scattering. The boundary brings about reflected and refracted waves; for dielectric media, the reflected light radiation, in most cases, can be neglected, since it leads to small radiation amount dependent on the reflectivity mismatch. In contrast, for an elastic medium at the boundary with a vacuum, the total radiation is returned back; thus the boundary effect turns out to be much stronger for an elastic waves as compared with optics. 
The results obtained permit us to conclude that under similar conditions, multiply scattered elastic waves travel much longer on an acoustic path, than the light waves, over relevant extinction scales.

For the scalar model considered presently the effect of boundary is simply accounted for due to the fact that the boundary-induced term of the Green's function long-range asymptotics takes the form of a spherical wave generated by a single virtual mirror image source. For a more sophisticated problem of elastic wave multiple scattering, one is to take into account the tensorial character of the Green's function. The boundary-induced term specifically describes, in the far field zone, non-trivial transformations at the vacuum-medium boundary of three incident elastic wave modes into three reflected ones as inputs of different mirror image sources for different types of incident-reflected modes pairs. Near the boundary, above all, the Rayleigh surface waves' input must also be taken into account. We hope that the simulations performed here can be generalized, accounting for all elastic wave modes.

\section{Acknowledgments}

This work was supported partially by the Russian Foundation for Basic Research (Grant No. 13-02-01255).

\section{References}

[1] Ishimaru A. Wave Propagation and Scattering in Random Media. IEEE Press Series on Electromagnetic Wave Theory. Wiley, New York, 1999.

[2] Sir W. Thomson. Note on the integration of the equations of equilibrium of an elastic solid. Cambridge and Dublin Mathematical Journal, 1848, 3, P. 87-89.

[3] Sir G. G. Stokes. On the theories of the internal friction of fluids in motion, and of the equilibrium and motion of elastic solids. Trans. Cambridge Phil. Soc., 1849, 8, P. 287-319.

[4] Aki K. and Richards P. G.. Quantitative Seismology: Theory and Methods. University Science Books, Sausalito, CA, 2nd edition, 2002.

[5] Felsen L. B. and Marcuvitz N. Radiation and Scattering of Waves. IEEE Press Series on Electromagnetic Wave Theory. John Wiley\& Sons, New York, 2003.

[6] Kachanov M. L., Shafiro B., and Tsukrov I. Handbook of Elasticity Solutions. Kluwer Academic Publisher, Dordrecht, 2003.

[7] Aki K. Analysis of the seismic coda of local earthquakes as scattered waves. Journal of Geophysical Research, 1969, 74(2), P. 615-631.

[8] Herraiz M. and Espinosa A. F. Coda waves: A review. Pure and Applied Geophysics, 1987, 125(4), P. 499-577.

[9] Snieder R. Coda wave interferometry and the equilibration of energy in elastic media. Phys. Rev. E, 2002, 66, P. 046615.

[10] Snieder R. Extracting the green's function from the correlation of coda waves: A derivation based on stationary phase. Phys. Rev. E, 2004, 69, P. 046610.

[11] Anache-Ménier D., van Tiggelen B. A., and Margerin L. Phase statistics of seismic coda waves. Phys. Rev. Lett., 2009, 102, P. 248501.

[12] van Tiggelen B. A. and Skipetrov S. E., editors. Wave Scattering in Complex Media: From Theory to Applications, volume 107 of NATO Science Series II: Mathematics, Physics and Chemistry, Kluwer Academic Publisher, London, 2003.

[13] van Rossum M. C. W. and Nieuwenhuizen Th. M. Multiple scattering of classical waves: microscopy, mesoscopy, and diffusion. Rev. Mod. Phys., 1999, 71, P. 313-371.

[14] Abubakirov I. R. and Gusev A.A. Estimation of scattering properties of lithosphere of kamchatka based on monte-carlo simulation of record envelope of a near earthquake. Physics of the Earth and Planetary Interiors, 1990, 64(1), P. 52-67.

[15] Sato H. Multiple isotropic scattering model including p-s conversions for the seismogram envelope formation. Geophysical Journal International, 1994, 117(2), P. 487-494. 
[16] Shapiro S. A. and Treitel S. Multiple scattering of seismic waves in multilayered structures. Physics of the Earth and Planetary Interiors, 1997, 104(1-3), P. 147-159.

[17] Trégourès N. P., Hennino R., Lacombe C., Shapiro N. M., Margerin L., Campillo M., and van Tiggelen B. A. Multiple scattering of seismic waves. Ultrasonics, 2002, 40(1-8), P. 269-274.

[18] Malcolm A. E., Scales J. A., and van Tiggelen B. A. Extracting the green function from diffuse, equipartitioned waves. Phys. Rev. E, 2004, 70, P. 015601.

[19] Trujilio L., Peniche F., and Jia X. Waves in Fluids and Solids, chapter 5. Multiple Scattering of Elastic Waves in Granular Media: Theory and Experiments, pages 127-152. Intech, Chanhai, China, 2011.

[20] Margerin L. Diffusion approximation with polarization and resonance effects for the modelling of seismic waves in strongly scattering small-scale media. Geophysical Journal International, 2013, 192(1), P. 326345.

[21] Hoshiba M. Simulation of multiple-scattered coda wave excitation based on the energy conservation law. Physics of the Earth and Planetary Interiors, 1991, 67(12), P. 123-136.

[22] Gusev A. A. and Abubakirov I. R. Vertical profile of effective turbidity reconstructed from broadening of incoherent body-wave pulses - I. General approach and the inversion procedure. Geophysical Journal International, 1999, 136(2), P. 295-308.

[23] Ryzhik L., Papanicolaou G., and Keller J. B.. Transport equations for elastic and other waves in random media. Wave Motion, 1996, 24(4), P. 327-370.

[24] Margerin L., Campillo M., and van Tiggelen B. A. Monte carlo simulation of multiple scattering of elastic waves. Journal of Geophysical Research, 2000, 105(B4), P. 7873-7892.

[25] Meglinski I. V., Kuzmin V. L., Churmakov D. Y., and Greenhalgh D. A. Monte carlo simulation of coherent effects in multiple scattering. Proceeding of the Royal Society A, 2005, 461(2053), P. 43-53.

[26] Landau L. D. and Lifshitz E. M. Theory of Elasticity. Butterworth-Heinemann, Oxford, 3rd edition, 2007.

[27] Jackson J. D. Classical Electrodynamics. Wiley, New York, 3rd. edition, 1998.

[28] Johnson L. R.. Green's function for Lamb's problem. Geophysical Journal of the Royal Astronomical Society, 1974, 37(1), P. 99-131.

[29] Lax M. and Nelson D. F.. Adventures in Green's land: Light scattering in. anisotropic media. In L. Mandel and Emil E. Wolf, editors, Proceedings of the Third Rochester Conference on Coherence and Quantum Optics, Plenum Press, New York, 1973, P. 415-445.

[30] Lax M. and Nelson D. F. Light scattering in crystals with surface corrections. In B. Bendow, V. M. Agranovich, and J. L. Birman, editors, Theory of Light Scattering in Condensed Media, Proc. of the First Joint USA-USSR Symp., Plenum Press, New York, 1976, P. 371-90.

[31] Val'kov A., Kuzmin V., Romanov V., Nikitina M., Kozhevnikov S., and Meglinski I. Field of a point source in a semi-infinite elastic medium. Waves in Random and Complex Media, 2012, 22(3), P. 423-434.

[32] Snieder R. Scattering: scattering and inverse scattering in pure and applied science, volume 1 of Scattering: Scattering and Inverse Scattering in Pure and Applied Science, chapter 1.7.1 - General Theory of Elastic Wave Scattering, Academic Press, London, 2002, P. 528-542.

[33] Maeda T., Sato H., and Nishimura T. Synthesis of coda wave envelopes in randomly inhomogeneous elastic media in a half-space: single scattering model including rayleigh waves. Geophysical Journal International, 2008, 172(1), P. 130-154.

[34] Devroye L. Non-Uniform Random Variate Generation. Springer, New York, 1986.

[35] Sato H., Fehler M. C., and Maeda T. Seismic Wave Propagation and Scattering in the Heterogeneous Earth. Springer, Berlin, 2nd edition, 2012.

[36] Marston P. L. Generalized optical theorem for scatterers having inversion symmetry: Applications to acoustic backscattering. J. Acoust. Soc. Am., 2001, 109(4), P. 1291-1295.

[37] Margerin L. and Sato H. Generalized optical theorems for the reconstruction of green's function of an inhomogeneous elastic medium. The Journal of the Acoustical Society of America, 2001, 130(6), P. 3674-3690. 